\title{
Abnormal Behavior Detection Based on Global Motion Orientation
}

\author{
Xuyan Ma ${ }^{1, a}$, Guomao Liang ${ }^{1, b}$, Wei $\mathrm{Yu}^{1, \mathrm{c}}$, Zhiyi $\mathrm{Qu} \mathrm{u}^{1, \mathrm{~d}}$ \\ ${ }^{1}$ School of Information Science \& Engineering, Lanzhou University, Lanzhou, China \\ amaxuyan198708@163.com, blianggm10@Izu.edu.cn, '626748450@qq.com, 'duzy@lzu.edu.cn
}

Keywords: abnormal behavior detection; global motion orientation; support vector machine (SVM).

\begin{abstract}
A novel approach is introduced in this paper to detect abnormal behavior based on global motion orientation. Compare to the normal behavior (walking, shaking hands etc.), abnormal behavior has different orientation. The method we introduced divides each frame into blocks, makes statistical analysis of the global motion direction histogram of all frame blocks and extracts characteristics. At last, behavior is detected with support vector machine (SVM). Experiment shows that the method proposed in the paper has certain robustness and can achieve real-time monitoring.
\end{abstract}

\section{Introduction}

With the growing concern of the social security problems, and the continuous development of science and technology, intelligent video surveillance system in public security is more and more important. And abnormal behavior detection is one of its important aspects. So what is abnormal behavior? Typically, in certain scenes (such as traffic junctions, banks, parking lots, shopping malls etc.), distinguished from the normal situation is called abnormal behavior (such as driving violations, robbery, burglary, hovering, suddenly accelerated, fire, explosion etc.). This paper takes fight abnormal behavior detection as an example.

A key step of abnormal behavior monitoring is feature extraction. Reference [3] directly analyses the principal component of all the frames' foreground segmentation figures to obtain the movement characteristics of a video segment. Due to the large amount of data image sequence using principal component analysis PCA (Principle component analysis), the computation this method needs is very large. In this paper, by calculating the global motion direction as the characteristics, the amount of computation can reduce effectively.

\section{Global Motion Direction}

The process of the algorithm is shown in Fig. 1.

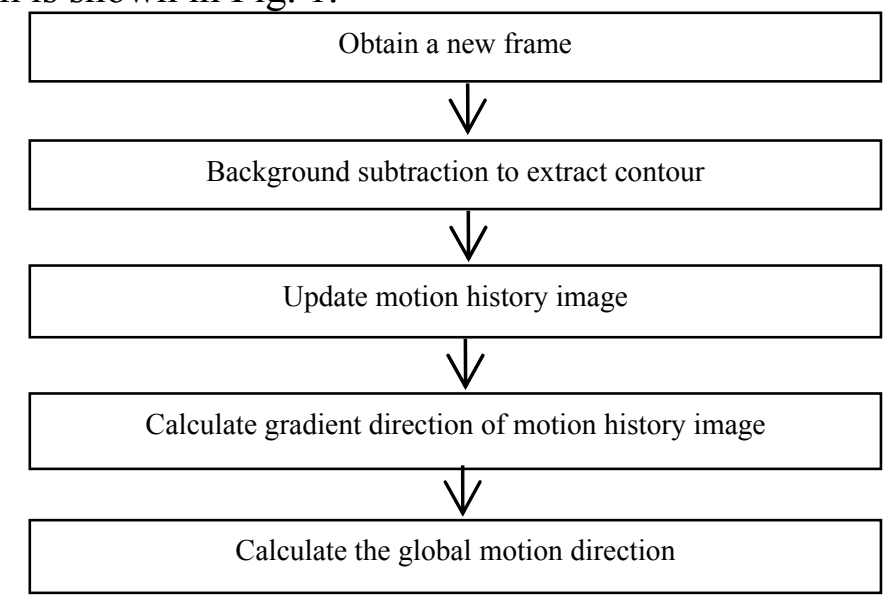

Figure1.The flowchart of calculating the global motion direction

Motion History Image. In this paper, we use the float motion history image (MHI) to couple the new value of the outline with a pixel timestamp (seconds, milliseconds). In [2] it is called tMHI (timed Motion History Image). We adopt the way of Eq.1 to update the motion history image: 


$$
{ }^{2} M I_{\delta}(x, y)= \begin{cases}\tau & \text { if current silhouette at }(x, y) \\ 0 & \text { else if } \operatorname{tMHI}_{\delta}(x, y)<(\tau-\delta)\end{cases}
$$

Where $\tau$ is the current timestamp, $\delta$ is the maximum duration of the motion tracking. That is in tMHI moving pixels are set as the current timestamp, while the pixels whose movement occurred earlier will be cleared (Fig. 2b).

Gradient Direction of the Motion History Image. By calculating the tMHI gradient direction in Fig. 2b, we can get the object motion direction. The tMHI gradient direction in $\mathrm{X}$, Y direction can effectively calculated by using a separate Sobel operator. The calculation formula of the gradient direction of each pixel is shown in Eq.2:

$$
\phi(x, y)=\arctan \frac{F_{y}(x, y)}{F_{x}(x, y)}
$$

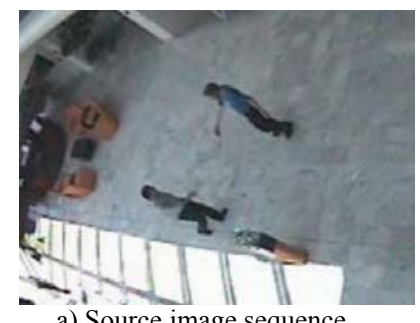

a) Source image sequence

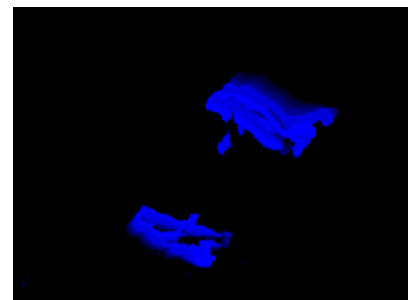

b) Motion history image

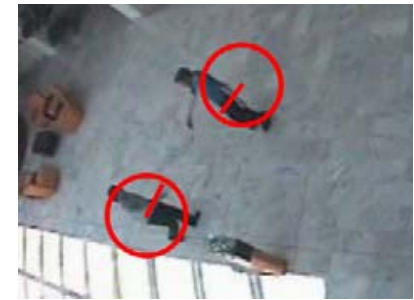

c) Global motion direction

Figure2.The calculation of the global motion direction

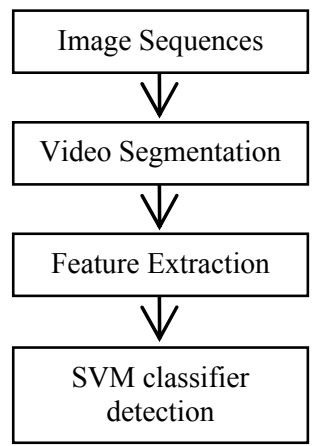

Figure3.The process of detecting the fight abnormal behavior

Global Motion Direction. When calculating the global motion direction, in order to highlight the present movement, the normal tMHI value weight should be considered. As shown in Eq. 3:

$$
\bar{\phi}=\phi_{\text {ref }}+\frac{\sum_{x, y} \operatorname{angDiff}\left(\phi(x, y), \phi_{\text {ref }}\right) \times \operatorname{norm}\left(\tau, \delta, \text { MHII }_{\delta}(x, y)\right)}{\sum_{x, y} \operatorname{norm}\left(\tau, \delta, \operatorname{tMHI}_{\delta}(x, y)\right.}
$$

Where $\bar{\phi}$ is the global motion direction; $\phi_{\text {ref }}$ is the basic reference angle; $\phi(x, y)$ is the mapping

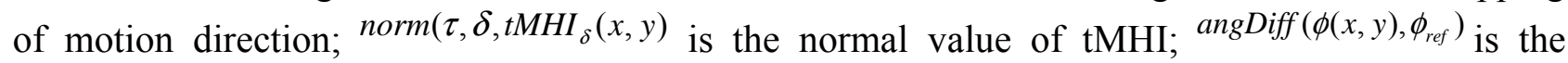
smallest signed angle difference by subtracting the reference angle. Fig. $2 \mathrm{c}$ is the global motion direction of two walking people.

\section{Abnormal Behavior Detection}

In this paper, the detection process of abnormal behavior (Fig. 3) includes three steps: video segmentation, feature extraction and the detection of SVM classifier.

Segment the input video. In order to make sure that each video segment contains a separate action, we use the method of [1] to fix the window length as 4 seconds (100 frames). Although this segmentation method can not guarantee each video segment corresponds to a complete action, 4 seconds can often provide sufficient information to determine the action attribute of a video segment. For example, in public places, 4 seconds can complete a handshake action. This interval overlapping window can divide the video into fixed-length video segment, and sets the beginning frame intervals of the adjacent video segment as 10 frames. 
Feature Extraction. After the video segmentation, the next step is to extract the characteristics of each segment. Divide each frame into blocks, and then calculate the global motion directions in all blocks. Histogram statistics for all blocks in the direction of motion in the video segment, the value of the center of each block between $[0-2 \pi]$ can be obtained uniformly. The segment block can neither too large nor too small. Too small block is easily affected by the noise; too large block is easy to lose useful detail information. Test for $384 * 288$ video frames, the block is taking as $24 *$ 24. Compute the histograms of fight, single people walking, people walking and handshake four acts respectively (Fig. 4). Normalized histogram is shown in Fig. 5.

SVM Classifier Detection. SVM is a learning method based on the structural risk minimization criteria. SVM solving is converted into the solving of quadratic programming problems at last. So SVM solution is the globally unique optimal solution. SVM exhibits many unique advantages in solving small sample, nonlinear and high dimensional pattern recognition problem and has achieved good results in pattern recognition, function approximation and probability density estimation. Therefore, this paper uses SVM abnormal behavior detection training.
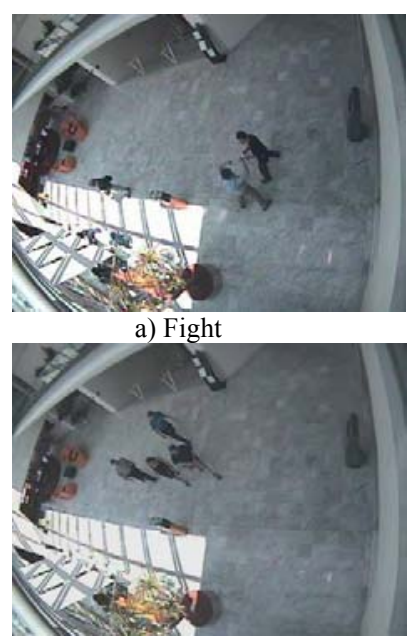

c) People walking

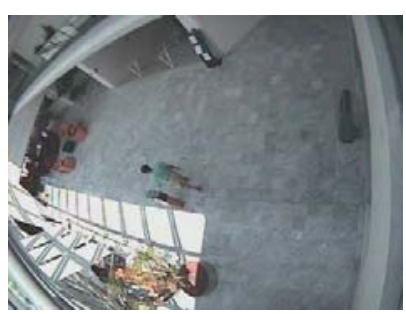

b) Single person walking

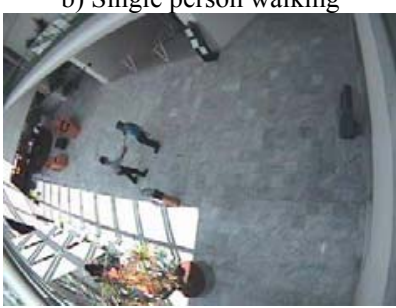

d) Handshake

Figure4.Behavior examples

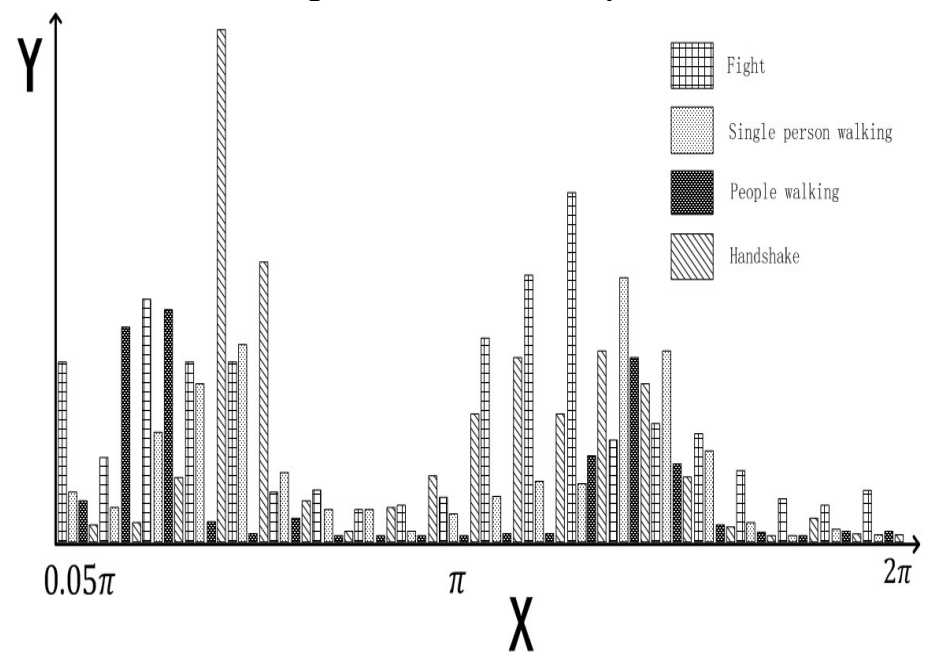

Figure5.The normalized histogram of movement direction of different behaviors ( $\mathrm{X}$ represents the center of each block, Y represents proportion.)

Fig. 5 shows the differences of movement direction histogram between the abnormal and the normal behaviors. In this paper we extract motion direction as the characteristic of the SVM classifier. Fight which is an abnormal behavior sample is classified as 1 and normal behavior sample is classified as - 1 . Extract the feature from movement direction of the histogram to get the sample set $\left\{\left(x_{i}, y_{i}\right) \mid x_{i} \in R^{N}, y_{i}= \pm 1, i=1,2 \cdots, n\right\}$ ( $\mathrm{N}$ is the number of the blocks). Use this sample to train SVM, and take the kernel function as radial basis function, as Eq.4 shows:

$$
K(x, y)=\exp \left(-\gamma\|x-y\|^{2}\right), \gamma>0
$$


Where $\gamma$ is a correction parameter of the nuclear function $K(x, y)$. For the unknown sample, the sample is a fight abnormal behavior when the discriminator function outputs 1 and a normal behavior when output is -1 . Through experiments, the correction parameter of the nuclear function $\gamma=10$.

\section{The Experimental Results}

In the training set, samples are video segments of fight, single people walking, people walking, handshake, a total of 367.Negative samples are fight video segments, a total of 78 samples (Fig. 4). The test sets are 200 positive samples and 60 negative samples. Table 1 shows the test results with different number of blocks.

TABLE I. $\quad$ THE TEST RESULTS WITH DIFFERENT NUMBER OF BLOCKS

\begin{tabular}{|c|c|c|c|c|c|c|c|}
\hline $\begin{array}{c}\text { The number of } \\
\text { blocks }\end{array}$ & 15 & 20 & 25 & 30 & 35 & 45 & 45 \\
\hline $\begin{array}{c}\text { Normal behavior } \\
\text { detection rate }\end{array}$ & 0.89 & 0.91 & 0.91 & 0.94 & 0.92 & 0.89 & 0.88 \\
\hline $\begin{array}{c}\text { Abnormal behavior } \\
\text { detection rate }\end{array}$ & 0.73 & 0.75 & 0.79 & 0.80 & 0.77 & 0.76 & 0.72 \\
\hline
\end{tabular}

As shown in the table, different numbers of blocks result in different test results. Normal and abnormal behaviors detection rate reaches the highest value when the block number is 30 . The number of blocks will affect the description of the characteristics. The fewer the number of blocks, then the behavior description more rough, relatively easy to extract the common characteristics among different behaviors. The more the number of blocks, then the behavior describes more details and easier to be distinguished from different behavior details. Therefore the number of blocks should be choice reasonably to make it most effective to distinguish normal and abnormal behaviors.

\section{Conclusion}

This paper describes an algorithm of abnormal behavior detection based on global motion direction. Experiments show that the method can detect fight abnormal behavior in certain places such as corridors, with certain robustness. Next, we can detect the abnormal behavior of cataplexy to achieve intelligent supervision and management of the bedroom or wards for the elderly. But when detect moving objects far away from the camera; the detection result is not satisfactory. In future studies, multi-camera applications should be considered.

\section{References}

[1] HU Zhi-Lan, JIANG Fan: Anomaly Detection Based on Motion Direction, ACTA AUTOMATICA SINICA, 2008, 34(11): 1348-1357.

[2] Gary R. Bradski, James Davis: Motion Segmentation and Pose Recognition with Motion History Gradients, Machine Vision and Application, 2002, 13(7): 174-184.

[3] Kyoko SUDO, Tatsuya Osawa, Detecting the degree of anomaly in security video sequence, MVA2007 IAPR Conference on Machine Vision Applications, Tokyo, Japan: 2007.

[4] Bian Zhao-Qi, Zhang Xue-Gong, Pattern Recognition. Beijing: Tsinghua University Press 2002.

[5] The video sequence come from the EC Funded CAVIAR project/IST 2001 37540, found at URL: http://homepages.inf.ed.ac.uk/rbf/CAVIAR/. 